\title{
Applying Neural Network Architecture for Inverse Kinematics Problem in Robotics
}

\author{
Bassam Daya, Shadi Khawandi, Mohamed Akoum
}

Institute of Technology, Lebanese University, Saida, Lebanon.

Email: b_daya@ul.edu.lb

Received November $12^{\text {th }}, 2009$; revised December $15^{\text {th }}, 2009$; accepted December $22^{\text {nd }}, 2009$.

\begin{abstract}
One of the most important problems in robot kinematics and control is, finding the solution of Inverse Kinematics. Inverse kinematics computation has been one of the main problems in robotics research. As the Complexity of robot increases, obtaining the inverse kinematics is difficult and computationally expensive. Traditional methods such as geometric, iterative and algebraic are inadequate if the joint structure of the manipulator is more complex. As alternative approaches, neural networks and optimal search methods have been widely used for inverse kinematics modeling and control in robotics This paper proposes neural network architecture that consists of 6 sub-neural networks to solve the inverse kinematics problem for robotics manipulators with 2 or higher degrees of freedom. The neural networks utilized are multi-layered perceptron (MLP) with a back-propagation training algorithm. This approach will reduce the complexity of the algorithm and calculation (matrix inversion) faced when using the Inverse Geometric Models implementation (IGM) in robotics. The obtained results are presented and analyzed in order to prove the efficiency of the proposed approach.
\end{abstract}

Keywords: Inverse Geometric Model, Neural Network, Multi-Layered Perceptron, Robotic System, Arm

\section{Introduction}

The task of calculating all of the joint angles that would result in a specific position/orientation of an end-effector of a robot arm is called the inverse kinematics problem. In the recent years, the robot control problem has received considerable attention due to its complexity. Inverse kinematics modeling has been one of the main problems in robotics research, there has been a lot of research on the use of neural networks for control The most popular method for controlling robotic arms [1-5].

In inverse kinematics learning, the complexity is in the geometric and non linear equations (trigonometric equations) and in the matrix inversion, this in addition to some other difficulties faced in inverse kinematics like having multiple solutions. The traditional mathematical solutions for inverse kinematics problem, such as geometric, iterative and algebraic, may not lead always to physical solutions. When the number of manipulator degrees of freedom increases, and structural flexibility is included, analytical modeling becomes almost impossible. A modular neural network architecture was proposed by Jacobs et al. and has been used by many researches [2,3,5,6].

However, the input-output relation of their networks is continuous and the learning method of them is not sufficient for the non-linearity of the kinematics system of the robot arm.

This paper proposes neural network architecture for inverse kinematics learning. The proposed approach consists of 6 sub-neural networks. The neural networks utilized are multi-layered perceptron (MLP) with a backpropagation training algorithm. They are trained with end-effector position and joint angles.

In the sections that follow, we explain the inverse kinematics problem, and then we propose our neural network approach; we present and analyze the results in order to prove that neural networks provide a simple and effective way to both model the manipulator inverse kinematics and circumvent the problems associated with algorithmic solution methods.

The proposed approach is presented as a strategy that could be reused and implemented to solve the inverse kinematics problems faced in robotics with highest degrees of freedom. The basics of this strategy are explained in details in the sections that follow. 


\section{Inverse Kinematics Problem}

Inverse kinematics computation has been one of the main problems in robotics research. This problem is generally more complex for robotics manipulators that are redundant or with high degrees of freedom. Robot kinematics is the study of the motion (kinematics) of robots. They are mainly of the following two types: forward kinematics and inverse kinematics. Forward kinematics is also known as direct kinematics. In forward kinematics, the length of each link and the angle of each joint are given and we have to calculate the position of any point in the work volume of the robot. In inverse kinematics, the length of each link and position of the point in work volume is given and we have to calculate the angle of each join. In this section, we present the inverse kinematics problem.

\subsection{Inverse Position Kinematics and IGM}

The inverse position kinematics (IPK) solves the following problem: "Given the desired position of the robot's hand; what must be the angles at the robot joints?" In contrast to the forward problem, the solution of the inverse problem is not always unique: The same end effector's pose can be reached in several configurations, corresponding to distinct joint position vectors. The conversion of the position and orientation of a robot manipulator end-effector from Cartesian space to joint space is called inverse kinematics problem. For any position in the $\mathrm{X}-\mathrm{Y}$ plane for a 2R robot, there is a possibility of 2 solutions for any given point. This is due to the fact that there are 2 configurations that might be possible to reach the desired point as Figure 1.

The math involved in solving the Inverse Kinematics problem requires some background in linear algebra, specifically in the anatomy and application of transformation matrices.

Therefore, an immediate attempt to solve the inverse kinematics problem would be by inverting forward kinematics equations.

Let's illustrate how to solve the inverse kinematics problem for robot manipulators on a simple example. Figure 2 shows a simple planar robot with two arms. The underlying degrees of freedom of this robot are the two angles dictating the rotation of the arms. These are labeled in Figure 2 as $\theta_{1}$ and $\theta_{2}$. The inverse kinematics question in this case would be: What are the values for the degrees of freedom so that the end effector of this robot (the tip of the last arm) lies at position $(\mathrm{x}, \mathrm{y})$ in the two-dimensional Cartesian space? One straightforward approach to solving the problem is to try to write down the forward kinematics equations that relate $(\mathrm{x}, \mathrm{y})$ to the two rotational degrees of freedom, then try to solve these equations. This solution, named IGM (Inverse Geometric Model) will give us an answer to the inverse kinematics problem for this robot. The calculation is presented in Figure 3.

As it can be seen in the example above, the solutions to

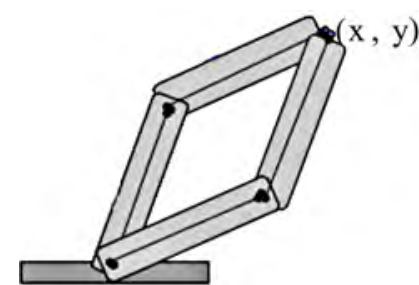

Figure 1. Two solutions depicted for the inverse kinematics problem

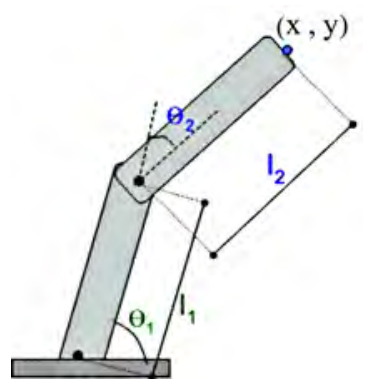

Figure 2. Steer end-effector (x, y) target position

$$
\begin{gathered}
x=l_{1} \cos \left(\theta_{1}\right)+l_{2} \cos \left(\theta_{1}+\theta_{2}\right) \\
y=l_{1} \sin \left(\theta_{1}\right)+l_{2} \sin \left(\theta_{1}+\theta_{2}\right) \\
x^{2}+y^{2}=l_{1}^{2}+l_{2}^{2}+2 l_{1} l_{2} \cos \left(\theta_{2}\right) \\
\cos \left(\theta_{2}\right)=\frac{x^{2}+y^{2}-l_{1}^{2}-l_{2}^{2}}{2 l_{1} l_{2}} \\
x=l_{1} \cos \left(\theta_{1}\right)+l_{2}\left(\cos \left(\theta_{1}\right) \cos \left(\theta_{2}\right)-\sin \left(\theta_{1}\right) \sin \left(\theta_{2}\right)\right) \\
x=\cos \left(\theta_{1}\right)\left(l_{1}+l_{2} \cos \left(\theta_{2}\right)\right)-\sin \left(\theta_{1}\right)\left(l_{2} \sin \left(\theta_{2}\right)\right) \\
y=\cos \left(\theta_{1}\right)\left(l_{2} \sin \left(\theta_{2}\right)\right)+\sin \left(\theta_{1}\right)\left(l_{1}+l_{2} \cos \left(\theta_{2}\right)\right) \\
\cos \left(\theta_{1}\right)=\frac{x+\sin \left(\theta_{1}\right) l_{2} \sin \left(\theta_{2}\right)}{l_{1}+l_{2} \cos \left(\theta_{2}\right)} \\
\sin \left(\theta_{1}\right)=\frac{\left(l_{1}+l_{2} \cos \left(\theta_{2}\right)\right) y-l_{2} \sin \left(\theta_{2}\right) x}{l_{1}^{2}+l_{2}^{2}+2 l_{1} l_{2} \cos \left(\theta_{2}\right)}
\end{gathered}
$$

Figure 3. Finding solutions from the forward kinematics equations

an inverse kinematics problem are not necessarily unique. In fact, as the number of degrees of freedom increases, so does the maximum number of solutions, as depicted in the figure. It is also possible for a problem to have no solution if the point on the robot cannot be brought to the target point in space at all.

While the above example offers equations that are easy to solve, general inverse kinematics problems require solving systems of nonlinear equations for which there are no general algorithms. Some inverse kinematics problems 
cannot be solved analytically. In robotics, it is sometimes possible to design systems to have solvable inverse kinematics, but in the general case, we must rely on approximation methods in order to keep the problem tractable, or, in some cases, even solvable.

It is known that there is a finite number of solutions to the inverse kinematics problem. There are, however, 3 types of solutions: complete analytical solution, numerical solutions and semi-analytical solution. In the first type, all of the joint variables are solved analytically according to given configuration data. In the second solution type, all of the joint variables are obtained iterative computational procedures. There are four disadvantages in these: 1) incorrect initial estimations, 2) before executing the inverse kinematics algorithms, convergence to the correct solution cannot be guaranteed, 3) multiple solutions are not known, 4)there is no solution, if the Jacobian matrix is singular. In the third type, some of the joint variables are determined analytically and some computed numerically. Disadvantage of numeric approaches to inverse kinematics problem is also heavy computational calculation and big computational time.

\section{Neural Network Approach}

The true power and advantage of neural networks lies in their ability to represent both linear and non-linear relationships and in their ability to learn these relationships directly from the data being modeled. Traditional linear models are simply inadequate when it comes to modeling data that contains non-linear characteristics. The most common neural network model is the multilayer perceptron (MLP). This type of neural network is known as a supervised network because it requires a desired output in order to learn. The goal of this type of network is to create a model that correctly maps the input to the output using historical data so that the model can then be used to produce the output when the desired output is unknown. A graphical representation of an MLP is shown in Figure 4.

The MLP and many other neural networks learn using an algorithm called back propagation. With back propagation, the input data is repeatedly presented to the neural network. With each presentation the output of the

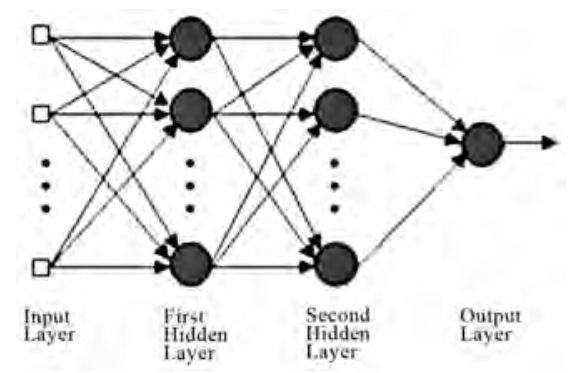

Figure 4. A two hidden layer multiplayer perceptron (MLP) neural network is compared to the desired output and an error is computed. This error is then fed back (back propagated) to the neural network and used to adjust the weights such that the error decreases with each iteration and the neural model gets closer and closer to producing the desired output. This process is known as "training".

As mentioned previously, for any position in the $\mathrm{X}-\mathrm{Y}$ plane for a $2 \mathrm{R}$ robot, there is a possibility of 2 solutions, so our approach started by implementing one sub-networks for each solution. For each network, we will try to obtain from the DGM model a series of $\mathrm{q}_{1}\left(\right.$ or $\left.\theta_{1}\right)$ and $\mathrm{q}_{2}\left(\right.$ or $\left.\theta_{2}\right)$ for a given position of the end-effector. The data training for the 2 sub-networks will be constructed as following:

- Neural Network NN1: $(x, y) \rightarrow\left(q_{1}, q_{2}\right)$;

$0 \quad \mathrm{q}_{1}$ between 0 and $2 \pi$, and $\mathrm{q}_{2}$ between 0 and $\pi$.

- Neural Network NN2: $(\mathrm{x}, \mathrm{y}) \rightarrow\left(\mathrm{q}_{1}, \mathrm{q}_{2}\right)$;

$0 \quad \mathrm{q}_{1}$ between 0 and $2 \pi$, but $\mathrm{q}_{2}$ between $-\pi$ and 0 .

As we are going to use DGM for generating our input parameters for the $\operatorname{MLP}\left(\mathrm{q}_{1}\right.$ and $\mathrm{q}_{2}$ ), for singular configurations, for the same point $\mathrm{X}$ and $\mathrm{Y}$, there are two solutions for it within the same aspect. For example, as shown in Figure 5, for 2 close positions of (x,y) we have big difference in $q_{1}$ values (first value of $q_{1}$ is close to 0 and the other $q_{1}$ is close to $2 \pi$ ). Thus, this will create a problem during training for our data.

So the above implementation (2 neural networks) leads to a big difficulty in the learning process. In order to solve this problem, we will use 4 Neural Networks (MLP); one for each quadrant.

By implementing 4 Neural Networks (MLP), we prevent two main problems: 1) no more problem to construct the training data for each aspect, and 2) no more problem in the learning phase (one output for one input).

For the error there will be two other Neural Networks:

- One for the Cartesian space (MLP5), used to classify whether the given position is within or not in the accessible region. The error will be representing whether the given solution is within the limitation of the robots.

- One for the joint space (MLP6) to respect the joint limits of our robot.

The approach will use the schematic of the neural network architecture given in Figure 6. The DGM model is used to handle the problem of identifying which MLP from the four MLPs is giving the right answer.

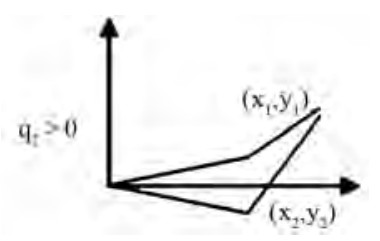

Figure 5. Two close positions for $(x, y)$ but too different values for $q_{1}(0$ and $2 \pi)$ and this is for the same Neural Network NN1 $\left(q_{2}>0\right)$ 


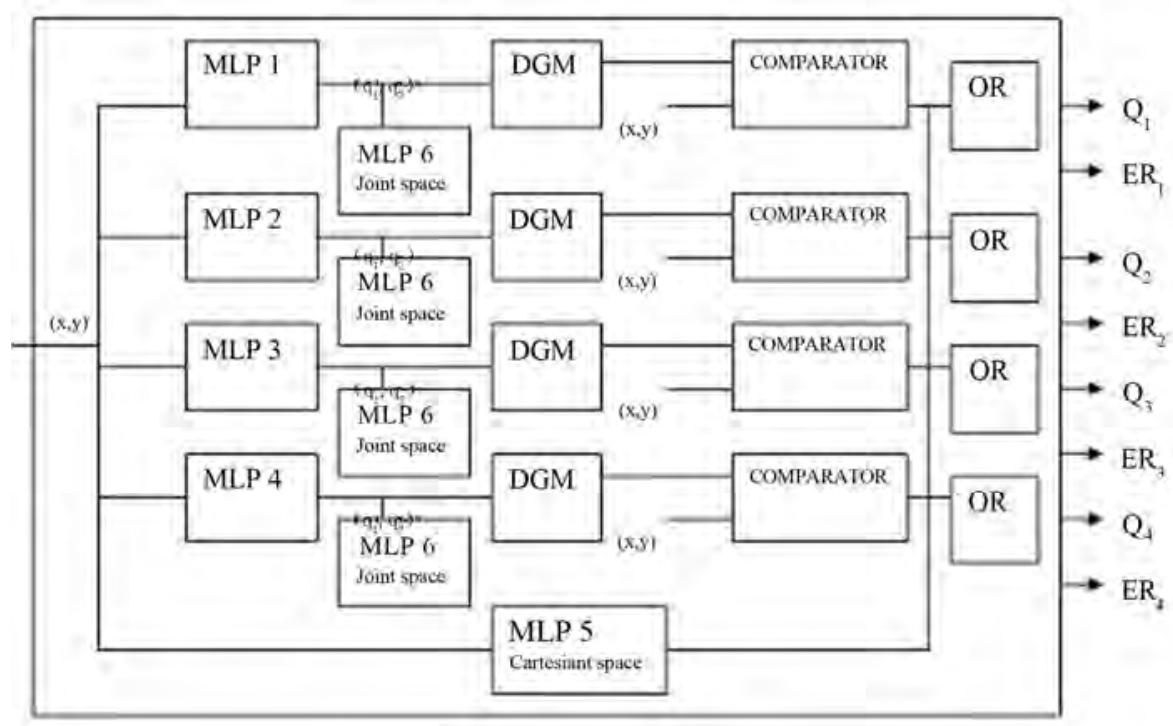

Figure 6. Multi-layer perceptron architecture using 4 MLPs (1 to 4 ) for the 4 quadrants, MLP5 for the Cartesian space and MLP6 for the joint space. Qi represent the output $\left(q_{1}, q_{2}\right)$ for $\operatorname{MLPi}(i=1$ to 4$)$ and ERi represent the error output ( if ERi $=0$ then Qi accepted; if $E R i=1$ then Qi rejected).

\section{Training, Experiments and Results}

In this section, we present the configuration and preparing of the neural network, and the experiments, with their results, executed in this approach.

We will use 6 MLPs to solve this problem. There will be 1 MLP for each quadrant of the joint space, as shown in Figure 7 , mainly for $q_{1}$ positive and $q_{2}$ positive, $q_{1}$ negative and $\mathrm{q}_{2}$ positive, $\mathrm{q}_{1}$ positive and $\mathrm{q}_{2}$ negative and lastly $\mathrm{q}_{1}$ negative and $\mathrm{q}_{2}$ negative. For the error there will be 2 other neural networks: one to classify whether the given position is within accessible region and one for the joint space.

\subsection{Creating MLP Network for IGM of 2R Planar Robot}

As mentioned above, for any position in the $\mathrm{X}-\mathrm{Y}$ plane for a $2 \mathrm{R}$ robot, there is a possibility of 2 solutions for any given point. The approach will try to obtain a series of $\mathrm{q}_{1}$ and $q_{2}$ for a given position of the end-effector in the $\mathrm{X}-\mathrm{Y}$ plane. This is due to the fact that there are 2 configurations that might be possible to reach the desired point.

For this MLP problem, we will be using 12 neurons for the first layer and 8 neurons for the second layer and 2 neurons for the output. However, the difference is that we will be using 6 MLPs to solve this problem. There will be 1 MLP for each quadrant of the joint space, as shown in Figure 7, mainly for $\left(\mathrm{q}_{1}>0\right.$ and $\left.\mathrm{q}_{2}>0\right),\left(\mathrm{q}_{1}>0\right.$ and $\mathrm{q}_{2}<$ $0)$, ( $\mathrm{q}_{1}<0$ and $\left.\mathrm{q}_{2}>0\right)$ and lastly $\left(\mathrm{q}_{1}<0\right.$ and $\left.\mathrm{q}_{2}<0\right)$.

This is done due to the fact that we are going to use DGM for generating our input parameters for the $\operatorname{MLP}\left(\mathrm{q}_{1}\right.$ and $\mathrm{q}_{2}$ ). For singular configurations, for the same point

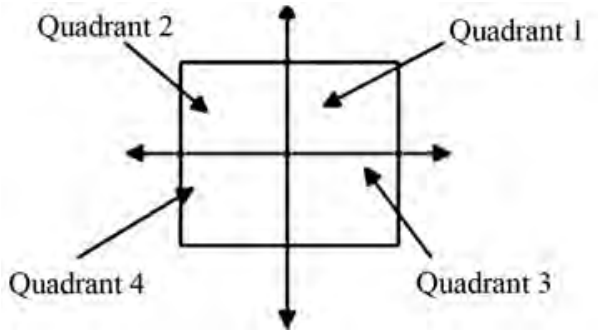

Figure 7. $\mathrm{X}$-Y plane in 4 quadrants

$\mathrm{X}$ and $\mathrm{Y}$, there are two solutions for it within the same aspect. For example, for $\mathrm{q}_{1}=\pi$ and $\mathrm{q}_{2}=-\pi$, they will have the same $X$ and $Y$ for any value of $q_{2}$. Thus, this will create a problem during training for our data. Thus, to solve this problem, we will be using one MLP for each quadrant of data. For the error there is perceptron used to classify whether the given $\mathrm{X}$ and $\mathrm{Y}$ is within accessible and non accessible region. The error will be representing whether the solution given is within the limitation of the robots. For example, if we give a point inside the non-accessible region of the working space, the error will be 1 and -1 for inside the accessible region.

For each quadrant, we created 10 variables for our target between $q_{1}=0$ to $\pi$ and equivalently for $q_{2}$. Then, we will use the Direct Geometry Model (DGM) function to generate the data training for our MLPs. The tansig function, used in our MLPs, will have its input from +3 to -3 and its output between +1 and -1 . The input and output for the MLP is then scaled according to the tansig function.

To achieve this, we multiply the input $\mathrm{x}$ by 2.5 such that, we will obtain $-3<\mathrm{x} \cdot 2.5<3$ (the same for the 4 MLPs). The other scaling is given in Table $\mathbf{1}$. 
Table 1. Scaling of the input $y$ and the outputs $q_{1}$ and $q_{2}$ for each MLP according to the tansig function

\begin{tabular}{cccc}
\hline No. & $\mathrm{q}_{1}$ & $\mathrm{q}_{2}$ & $\mathrm{Y}$ \\
MLP & $-1<\left(\mathrm{q}_{1} \cdot 2 / \pi\right)-1$ & $-1<\left(\mathrm{q}_{2} \cdot 2 / \pi\right)-1$ & $-3<(\mathrm{y} \cdot 5)-2<$ \\
\hline MLP1 & $<1$ & $<1$ & 3 \\
& $-1<\left(\mathrm{q}_{1} \cdot 2 / \pi\right)+1$ & $-1<\left(\mathrm{q}_{2} \cdot 2 / \pi-1<\right.$ & $-3<(\mathrm{y} \cdot 5)+2<$ \\
MLP2 & $<1$ & 1 & 3 \\
& $-1<\left(\mathrm{q}_{1} \cdot 2 / \pi\right)-1$ & $-1<\left(\mathrm{q}_{2} \cdot 2 / \pi\right)+1$ & $-3<(\mathrm{y} \cdot 5)-2<$ \\
MLP3 & $<1$ & $<1$ & 3 \\
& $-1<\left(\mathrm{q}_{1} \cdot 2 / \pi\right)+1$ & $-1<\left(\mathrm{q}_{2} \cdot 2 / \pi\right)-1$ & $-3<(\mathrm{y} \cdot 5)+2<$ \\
MLP4 & $<1$ & $<1$ & 3 \\
\hline
\end{tabular}

\subsubsection{The First MLP for the First Quadrant}

For the first quadrant, we created 10 variables for our target between $\mathrm{q}_{1}=0$ to $\pi$ and equivalently for $\mathrm{q}_{2}$. Then, we will use our DGM2R function to generate the input for our MLP. The input and output for the MLP is then scaled according to the tansig function. The tansig function will have its input from +3 to -3 and its output between +1 and -1 . To achieve this, will be dividing our output and multiplying our inputs with factors. The resulting input and output of for the MLP is shown in Figure 8.

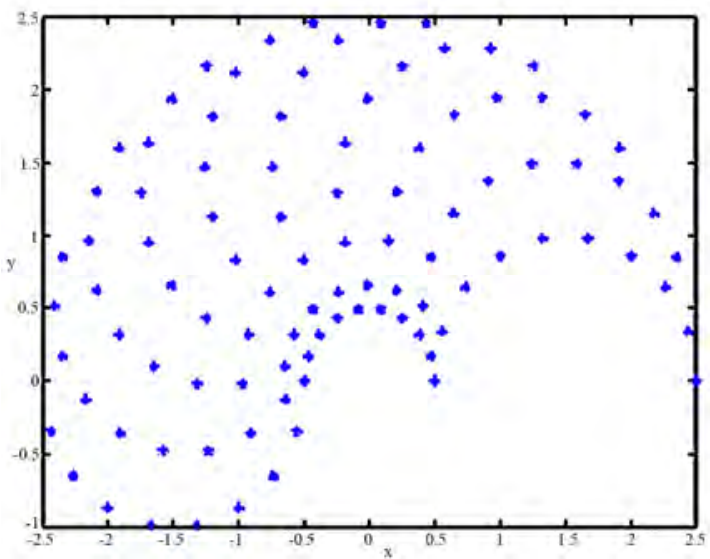

(a)

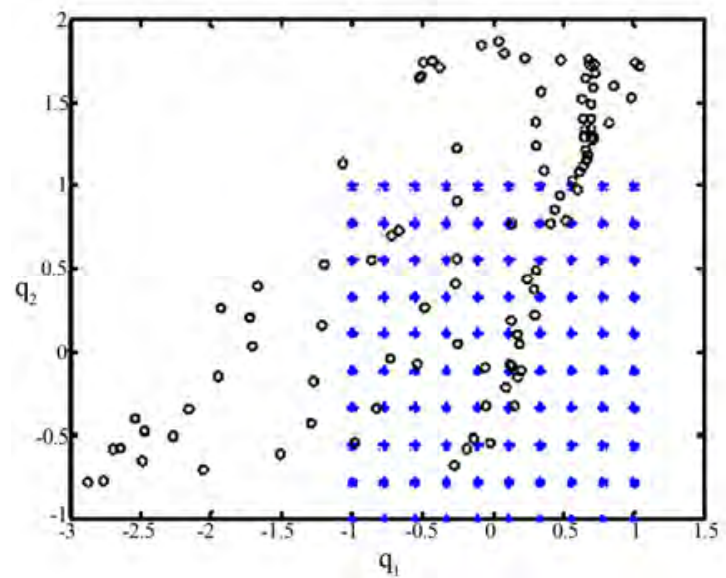

(b)

Figure 8. (a) Input for first quadrant MLP; (b) target output for first quadrant MLP
It is shown as well in Figure 8 that the initial result from our MLP prior to training. For our MLP, we will be using "trainlm" function since it is faster and more accurate in producing the result.

The MLP managed to produce accurate data with error of $1.74 \times 10^{-5}$ within 25 epochs. The result is then plotted back to our target. Figure 9 shows the result for the MLP restoring the data prior to scaling. From Figure 9, we know that our MLP has managed to produce quite an accurate result since the result of the MLP is pretty close to our target values.

For this MLP, we have used learning rate equal to 0.2. The performance of the MLP is shown in Figure 10.

\subsubsection{The Second MLP for the Second Quadrant}

In the second MLP for the second quadrant of the joint limit, we will do the same algorithm for training the MLP. We will input our data in the range of $q_{1}$ from 0 to -2.8 and $\mathrm{q}_{2}$ from 0 to $\pi$. We will then input our data to "tansig" transfer function. The inputs and outputs as well the initial output of our MLP are presented in Figure 11. Using the

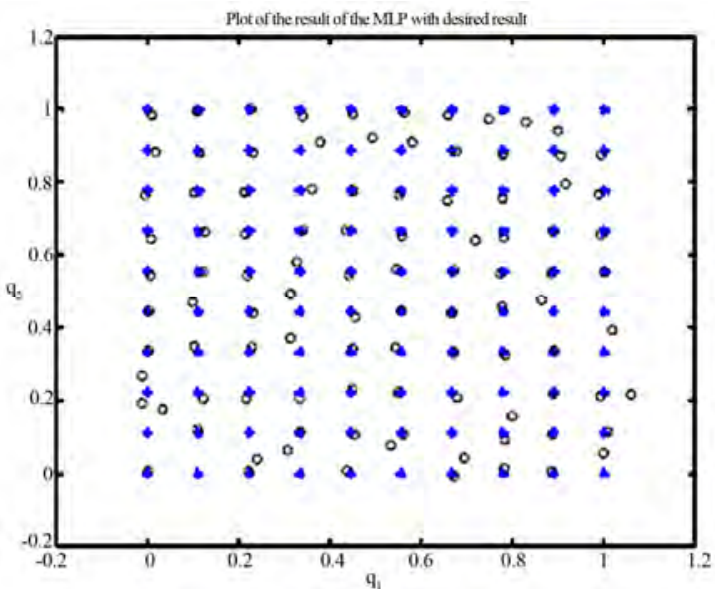

Figure 9. Result of the first quadrant MLP plotted onto the target data

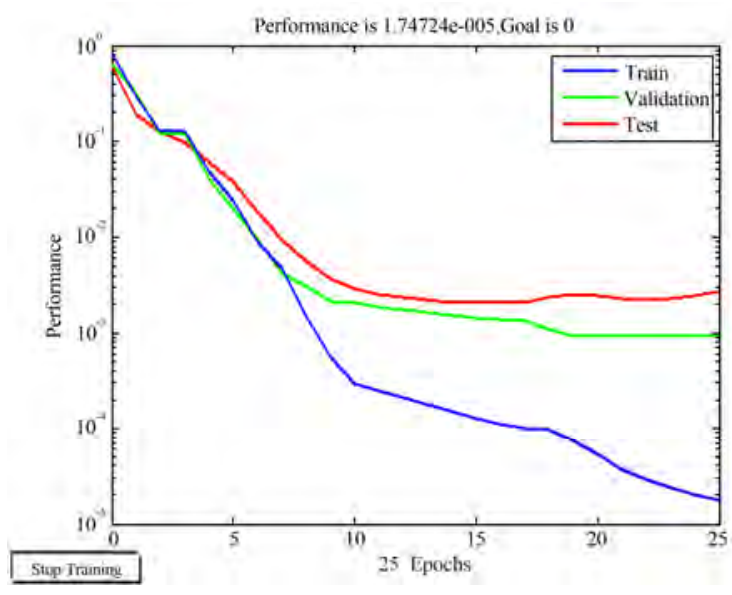

Figure 10. Performance result for this first MLP 
"trainlm" function, we managed to obtained accuracy of $1.38 \times 10^{-5}$ within 41 epochs.

The result of the MLP is presented in Figure 12. The result of the MLP presented is prior to rescaling back to the original data. For the third MLP we will be performing the similar operation by scaling the input and the output before inputting it to the MLP to be learnt.

\subsubsection{The Third MLP for the Third Quadrant}

For the third MLP, we are trying to generate result for $\mathrm{q}_{1}$ in the range of 0 and $\pi$ and $\mathrm{q}_{2}$ in the range of 0 to -2.8 . We are using -2.8 because of the requirement of the joint limit present in the system. The initial input and output of the system is presented in the following Figure 13.

After training our MLP for 15 epochs, we managed to get an error in performance of $4.64 \times 10^{-5}$. The plot of the result and the plot of the outputs are given in the following Figure 14.

\subsubsection{The Fourth MLP for the Fourth Quadrant}

For the last MLP to generate the result for IGM model, we

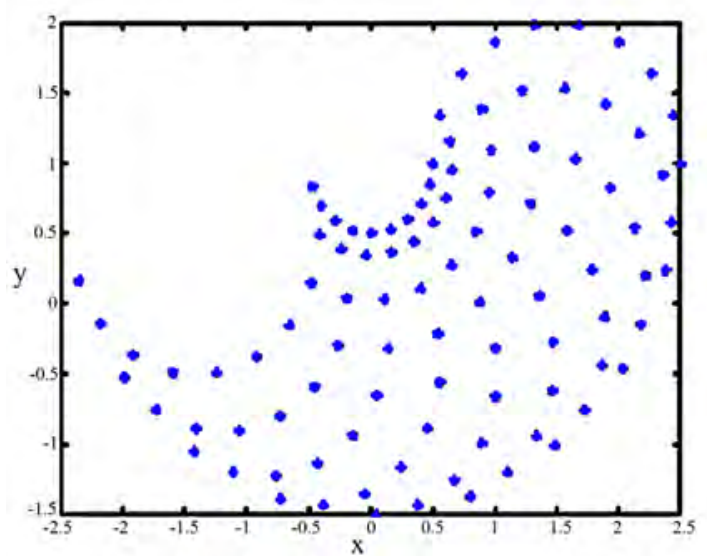

(a)

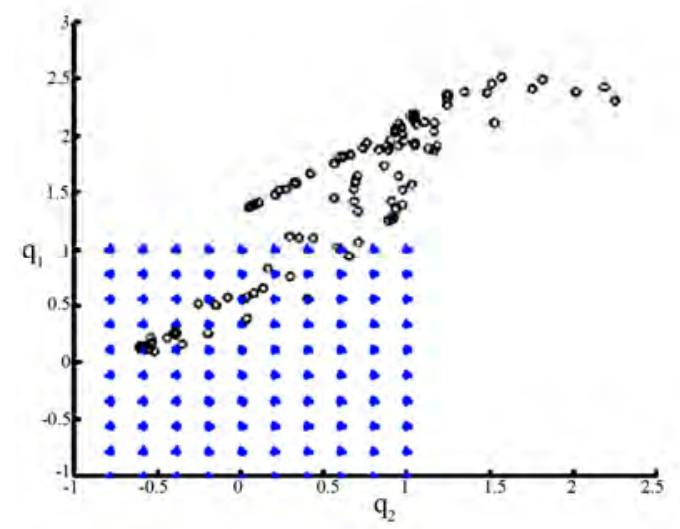

(b)

Figure 11. (a) Input data for the second quadrant MLP; (b) output of the second MLP plotted together with the desired result are trying to generate result for $\mathrm{q}_{1}$ in the range of 0 and -2.8 and $\mathrm{q}_{2}$ in the range of 0 to -2.8 . For the same reason, we are using -2.8 because of the requirement of the joint limit present in the system. The initial input and output of the system is presented in Figure 15. After training for 13 epochs, we managed to get an error of $5.24 \times 10^{-5}$ and the result of the MLP is plotted against the desired result. We can observe that the resulting points from the MLP are close to the desired target. The result of the MLP and the performance of the MLP are presented in Figure 16.

\subsubsection{The Fifth MLP}

The fifth MLP is designed to define the workspace of the robot. The robot workspace is a circle with an internal circle upon which the robot will not be able reach. Thus, there is a limitation to the area upon which the robot is able to access the area. When the given $\mathrm{x}$ and $\mathrm{y}$ is within the internal circle or outside the working circle as depicted in Figure 17, the error of the equation will be 1.

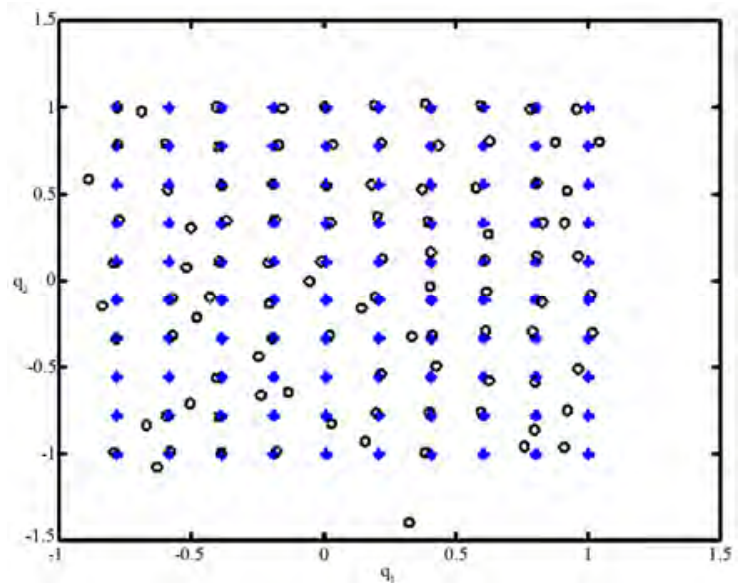

(a)

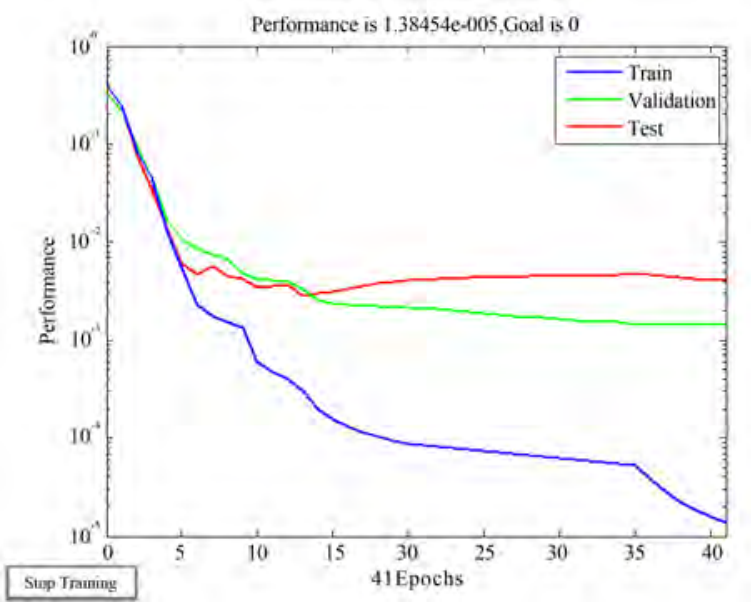

(b)

Figure 12. (a) Result of MLP plotted with the desired target; (b) Performance of MLP with trainml function 


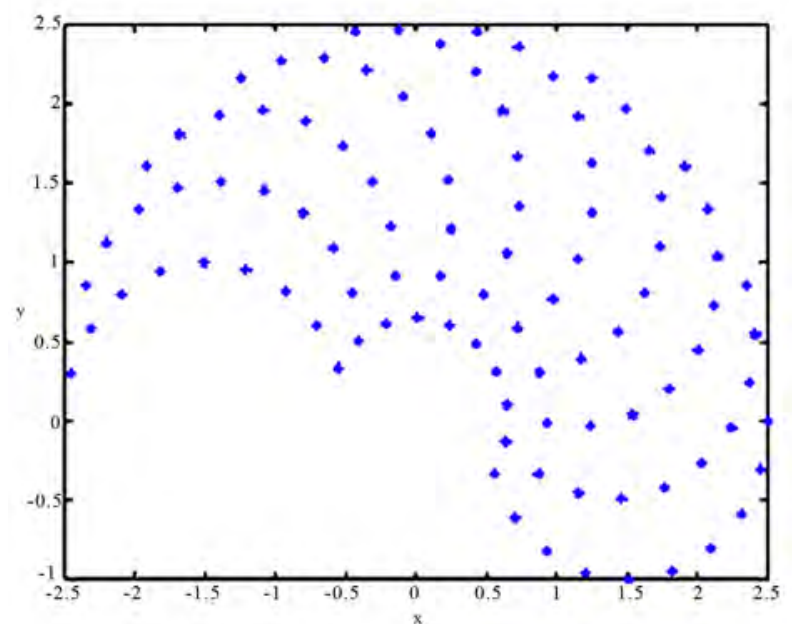

(a)

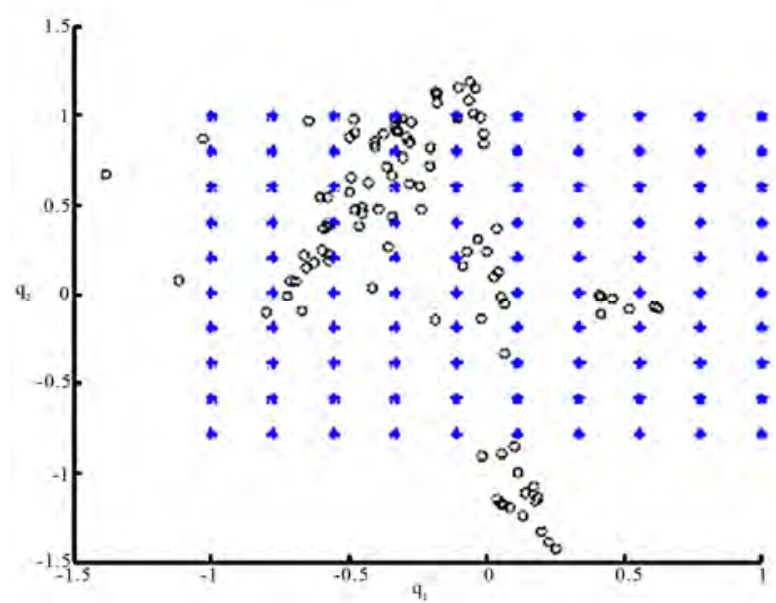

(b)

Figure 13. (a) Input for the $3^{\text {rd }}$ MLP; (b) output of the $3^{\text {rd }}$ MLP plotted with the desired result

In order to do this, we will find the relationship between the length of the robots arms to the radius of its working space. We know that the radius of the large cir cle is given by the formula $R=L_{1}+L_{2}$. Thus, we know that within the gray circle, $\left(R-L_{1}\right)^{2}=L_{2}^{2}$. Expanding the equation, we know that $R^{2}-2 L_{1} L_{2}+\left(L_{2}^{1}+L_{2}^{2}\right)=0$.

Thus, we notice that if the desired point is within the gray area, the value of the equation above will be less than 0 , and otherwise if the value of $R$ is smaller than $L_{1}$

$-\mathrm{L}_{2}$ or $\mathrm{R}$ is greater than $\mathrm{L}_{1}+\mathrm{L}_{2}$.

We have created 20 numbers of data of $X_{1}$ and $X_{2}$ for the input to the MLP.

Then, using these inputs, we calculate our desired target using the "error3" function using a notation that if error is 1 then the point is not inside working circle and if error is 0 then the robot is inside the working circle. The desired target is presented in Figure 18. Our result shows that

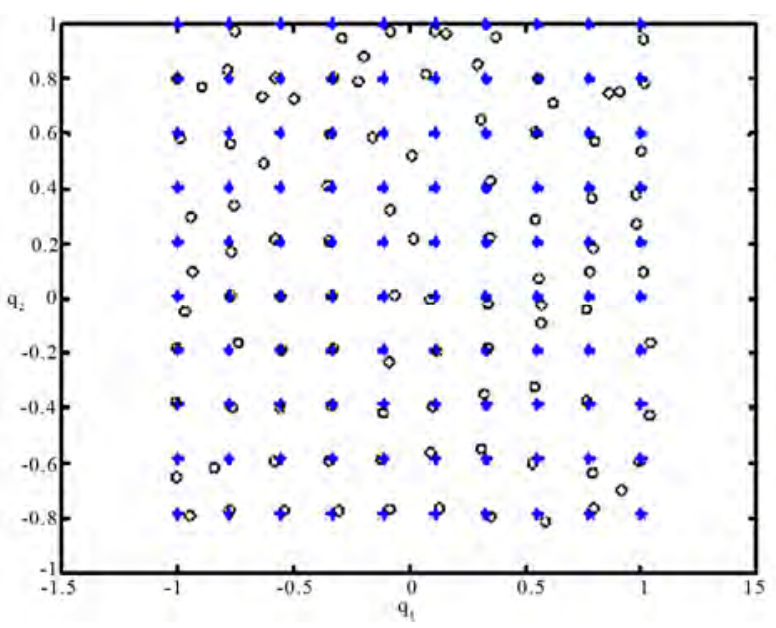

(a)

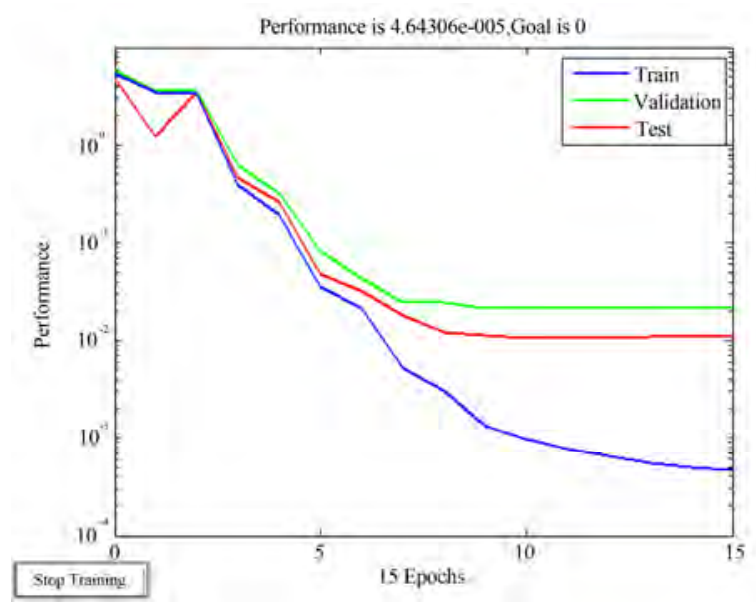

(b)

Figure 14. (a) Output of the result plotted together with the desired target of the $3^{\text {rd }}$ MLP; (b) Performance of the $3^{\text {rd }}$ MLP

the MLP has managed to classify the classes within 17 epochs with zero error. Thus, this error problem has been solved with only a single perceptron. The result of the MLP is presented in Figure 18.

The performance of the perceprton is shown in Figure 19.

\subsubsection{The Sixth MLP}

The last step of the classification is to categorize the resulting $\mathrm{Q}_{1}$ and $\mathrm{Q}_{2}$ into either [0 0], [0 1] or [ 10 0]. This means on the other hand, we need to classify the elements of angles in the $Q_{1}$ and $Q_{2}$. If we draw the boundary limit of the angles, we would be able to find a rectangular area(as shown in Figure 20). Certainly, we can apply the method of perceptron with 3 layers for implementing classifier arbitrary linearly limited areas (polyhedron).

Thus, if our MLP is having 4 neurons on the first layer and 1 neuron on the second layer and taking $Q_{1}$ and $Q_{2}$ as the input parameters for the neuron and output of 1 if $Q_{1}$ or 


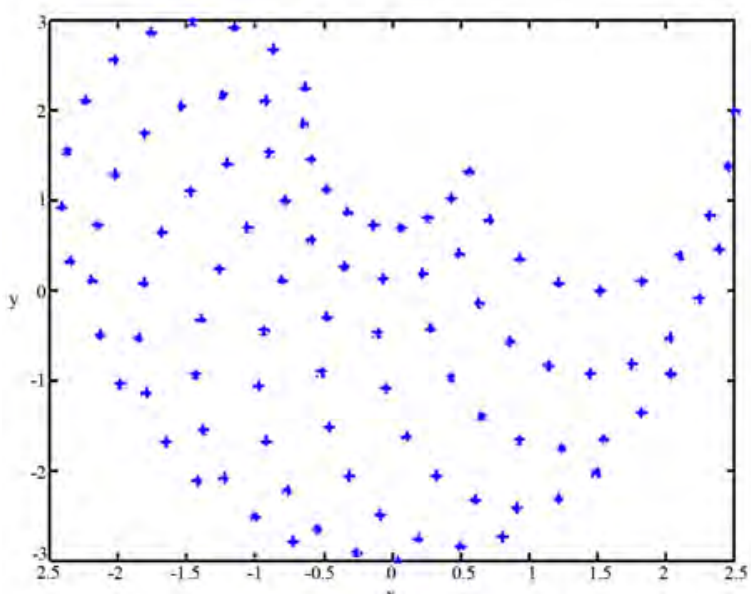

(a)

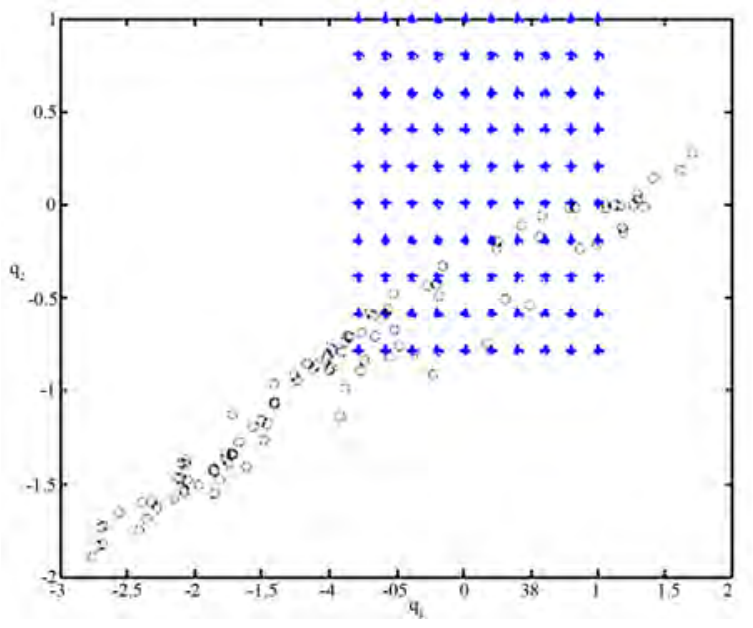

(b)

Figure 15. (a) Input to the $4^{\text {th }}$ MLP; (b) initial output of the $4^{\text {th }}$ MLP plotted together with the desired result

$\mathrm{Q}_{2}$ is within the grey area and -1 if it outside the gray area, we should be able to fully classify the problem.

The weights of our neurons are given as follows:

$$
W^{1}=\left[\begin{array}{ll}
1 & 0 \\
1 & 0 \\
0 & 1 \\
0 & 1
\end{array}\right] \text {, and } b^{1}=\left[\begin{array}{c}
-3 \\
2.8 \\
-3 \\
2.8
\end{array}\right]
$$

Marking the desired area (grey area), we obtained

\begin{tabular}{ccccc}
\hline & $\mathbf{g}_{1}$ & $\mathbf{g}_{2}$ & $\mathbf{g}_{3}$ & $\mathbf{g}_{4}$ \\
$\mathbf{G}_{1}$ & - & + & - & + \\
\hline
\end{tabular}

Thus, $W^{2}=\left[\begin{array}{llll}-1 & 1 & -1 & 1\end{array}\right]$, and $b^{2}=[-3]$.

From this weights and biases, our convention is :

- output $=1$ if the value of $Q$ is within the joint limit.

- output $=-1$ if the value of $\mathrm{Q}$ is outside the joint limit.

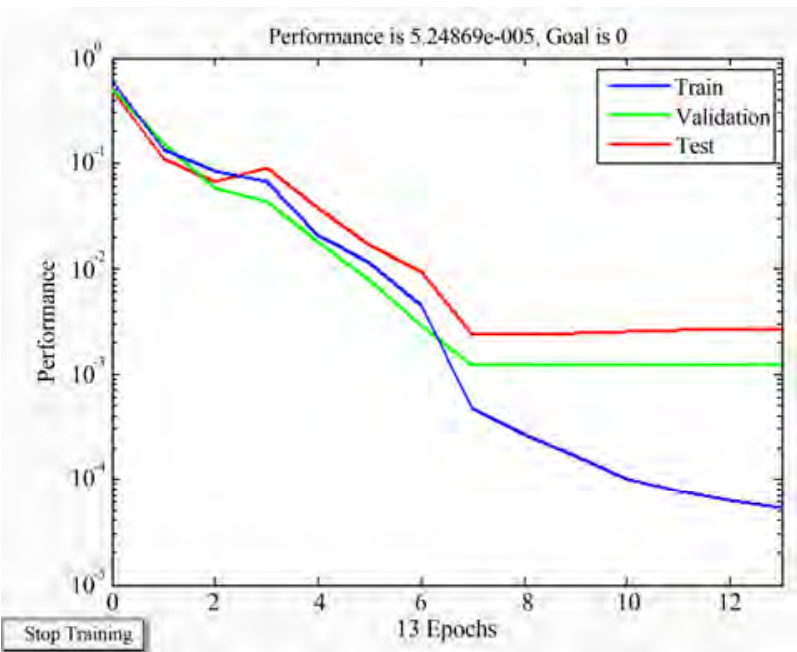

(a)

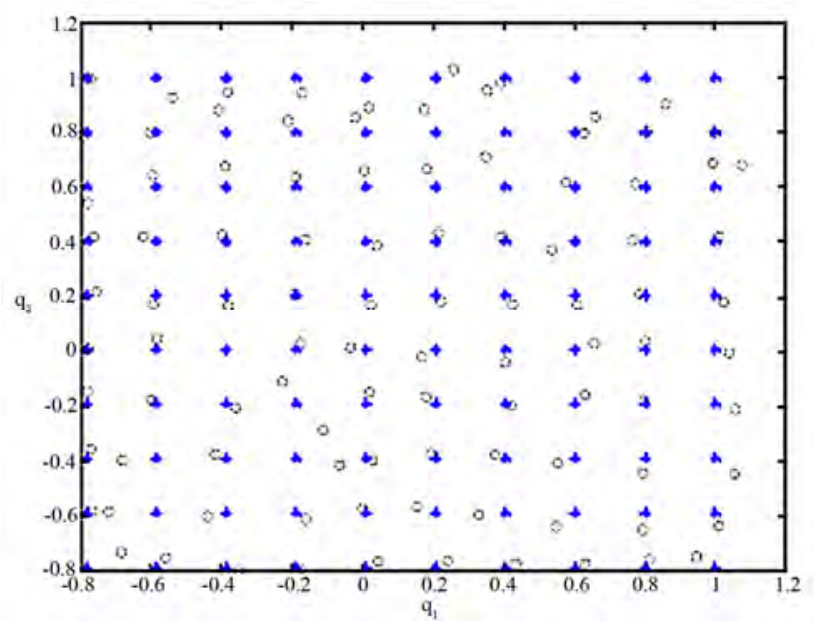

(b)

Figure 16. (a) Performance of the $4^{\text {th }}$ MLP; (b) Result of the $4^{\text {th }}$ MLP plotted together with the desired target

With these values, we can create a MLP network and we will be able to separate the two results perfectly. The result is shown in Figure 21. Lastly, the final step is to combine all the 6 MLP together in a program that we can use to generate the desired $\mathrm{Q}_{1}$ and $\mathrm{Q}_{2}$ and error. We will need to classify for the $\mathrm{y}$ of the input to our joint network. Initially, when the input is having $\mathrm{y}>0$, there are two solutions that are possible, which is $\mathrm{q}_{1}$ is positive and $\mathrm{q}_{2}$ is positive or negative. Thus, we have to choose quadrant 1 or 4 to obtain a correct result. Otherwise, when $y<0$, the two solutions that are possible are $q_{1}$ is negative and $q_{2}$ is positive or negative. After we have done the classification, then we can use our network to produce the desired result. The program will check whether the given $\mathrm{X}$ and $\mathrm{Y}$ is within the working circle. If it does not, then the error will be equal to [1 1 ] and the value of $Q_{1}$ and $Q_{2}$ will be of a null vector. On the other hand, if the point $\mathrm{X}, \mathrm{Y}$ is within 


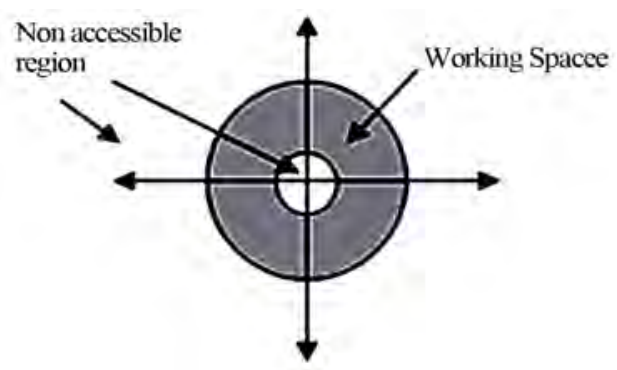

Figure 17. Working space of the robot

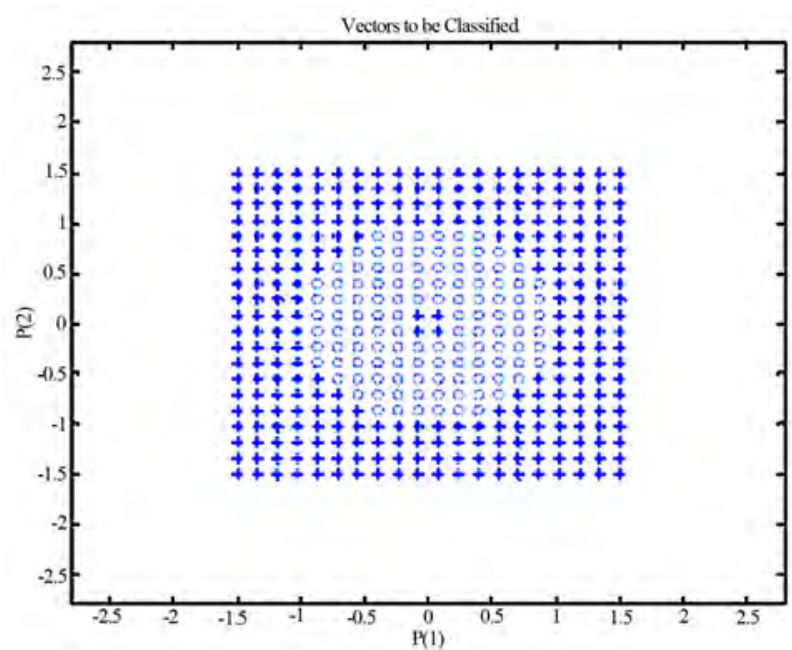

(a)

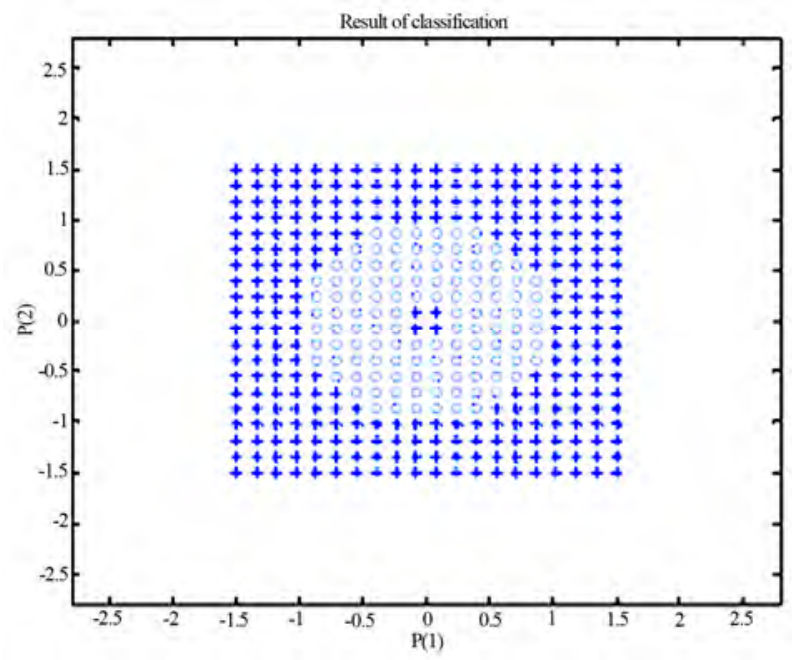

(b)

Figure 18. (a) Desired target result; (b) Result of the MLP

the circle, then it will input the $\mathrm{X}$ and $\mathrm{Y}$ according to the given area as described above. Then, the resulting result will be fed into the MLP 6 for determining whether the result is within the joint limit or not. If it does, then error will be equal to 0 and if it is not in the joint limit, then error will be equal to 1 .

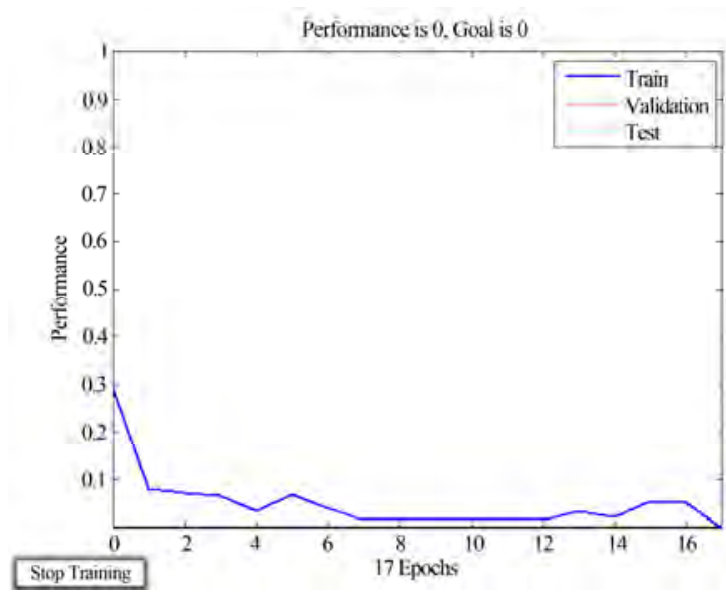

Figure 19. Performance of the perceprton

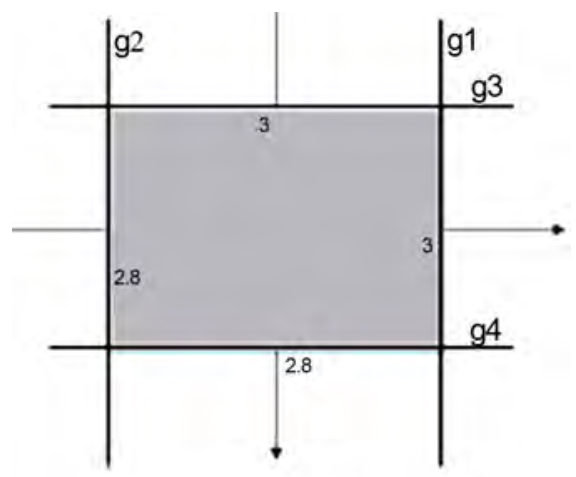

Figure 20. Classification to categorize $Q_{1}$ and $Q_{2}$

Testing the MLP with values of $X=\left[\begin{array}{ll}0.646 & -0.3196\end{array}\right]$ which corresponds to $q=\left[-\frac{\pi}{3} \frac{\pi}{2}\right]$, we obtained from our MLP,

$$
Q_{1}=\left[\begin{array}{ll}
1.0936 & 1.6016
\end{array}\right]
$$

and

$$
Q_{2}=\left[\begin{array}{ll}
2.2538 & -1.5618
\end{array}\right]
$$

Our optimum result for the $\mathrm{Q}_{1}$ and $\mathrm{Q}_{2}$ from the IGM model is

and

$$
Q_{1}=\left[\begin{array}{ll}
1.047 & 1.570
\end{array}\right]
$$

$$
Q_{2}=\left[\begin{array}{ll}
2.2232 & -1.5708
\end{array}\right]
$$

We know that the value from of MLP is pretty close to the real values of the IGM2R model. The error is [ 00$]$ and [0 0 0] respectively.

Testing the MLP with values of

$$
X=\left[\begin{array}{ll}
-0.3196 & -0.6464
\end{array}\right]
$$

which corresponds to $q=\left[\begin{array}{ll}-\frac{5 \pi}{6} & \frac{\pi}{2}\end{array}\right]$, we obtained from our MLP, 


$$
Q_{1}=\left[\begin{array}{ll}
-2.6856 & 1.8241
\end{array}\right]
$$

and

$$
Q_{2}=\left[\begin{array}{ll}
-1.4809 & -1.6115
\end{array}\right]
$$

Our optimum result for the $\mathrm{Q}_{1}$ and $\mathrm{Q}_{2}$ from the IGM model is

and

$$
Q_{1}=\left[\begin{array}{ll}
-2.6180 & 1.5708
\end{array}\right]
$$

$$
Q_{2}=\left[\begin{array}{ll}
-1.4420 & -1.5708
\end{array}\right]
$$

We know that the value from of MLP is pretty close to the real values of the IGM2R model. The error is $[00]$ and $\left[\begin{array}{ll}0 & 0] \\ \text { respectively. }\end{array}\right.$

\section{Conclusions}

In this paper, experimental results on the control of ro-

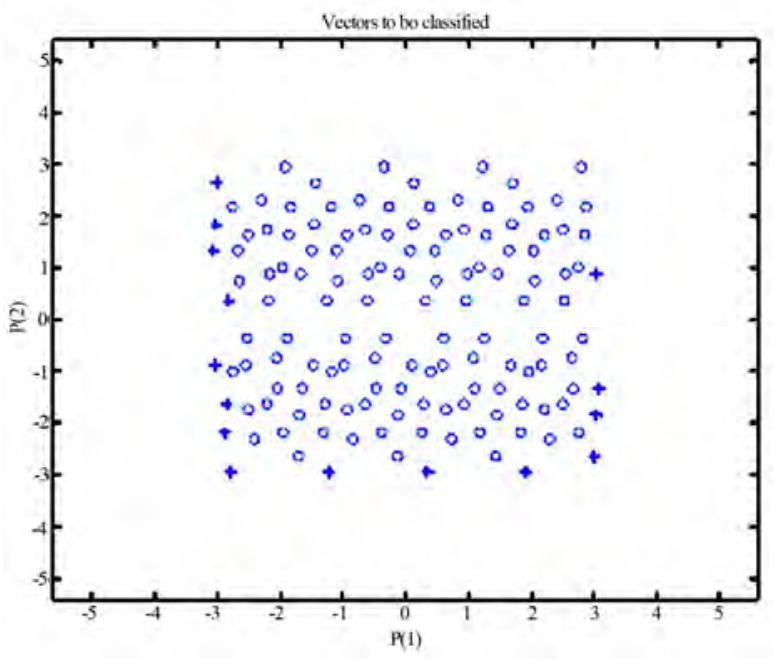

(a)

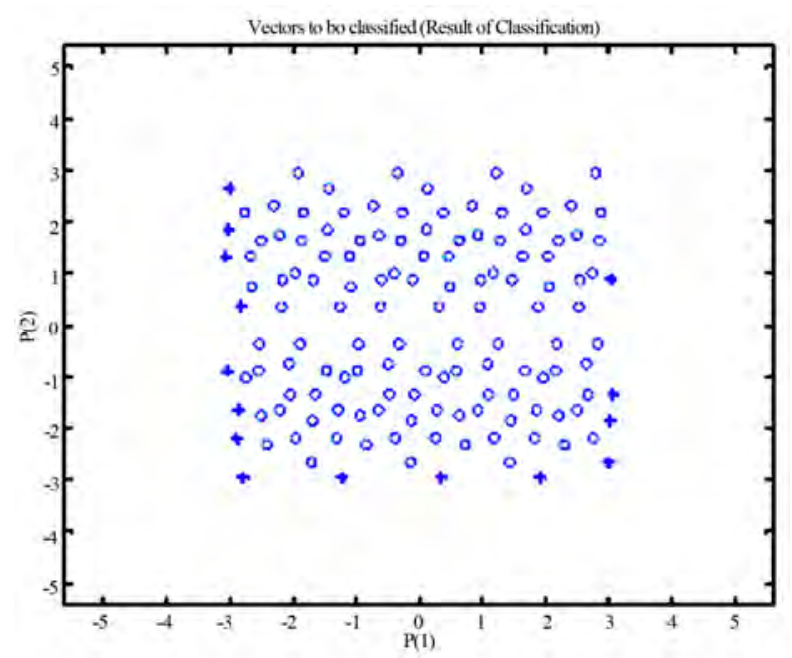

(b)

Figure 21. (a) Plot of the input $Q$ with the desired classification; (b) Plot of the input $Q$ with the result of classification botic manipulator using neural networks have been provided and it has been demonstrated that neural networks do indeed fulfill the promise of providing model-free learning controllers for robotic systems and provide an excellent alternative for the control of robotic manipulators.

Here, neural network model (MLP) solve the issues faced when the Inverse Geometric Model (IGM) is used, which requires no matrix inversion and iterates directly on the joint position, being thus suitable for on-line application and also preserving repeatability. In other words, Multilayer Networks are applied to the robot inverse kinematics problem. The networks are trained with endeffector position and joint angles. After training, performance is measured by having the network generate joint angles for arbitrary end-effector trajectories.

It is found that neural networks provide a simple and effective way to both model the manipulator inverse kinematics and circumvent the problems associated with algorithmic solution methods.

The proposed approach in the paper can be treated as a strategy to be followed for any other future work in the same domain. Mainly it can be implemented for robotics manipulators that are redundant or with high degrees of freedom. It is useful to mention that, based on the proposed approach; new research has been started lately for applying neural networks approach for 3R robotics.

\section{REFERENCES}

[1] B. Choi and C. Lawrence, "Inverse kinematics problem in robotics using neural networks,” National Aeronautics and Space Administration, Lewis Research Center, Cleveland, 1992.

[2] R. Köker, C. Öz, T. Çakar, and H. Ekiz , “A study of neural network based inverse kinematics solution for a three-joint robot," Robotics and Autonomous Systems, Vol. 49, pp. 227-234, 2004.

[3] L. Wei, H. Wang, and Y. Li , "A new solution for inverse kinematics of manipulator based on neural network," Machine Learning and Cybernetics, Vol. 2, pp. 1201-1203, 2003.

[4] J. Guo and V. Cherkassky, "A solution to the inverse kinematic problem in robotics using neural network processing”, International Joint Conference on Neural Networks, Vol. 2, pp. 299-304, 1989.

[5] D. Pham, M. Castellani, and A. Fahmy "Accountability learning the inverse kinematics of a robot manipulator using the Bees Algorithm," 6th IEEE International Conference on Industrial Informatics, pp. 493-498, 2008.

[6] E. Gallaf , "Multi-fingered robot hand optimal task force distribution: Neural inverse kinematics approach," Robotics and Autonomous Systems, Vol. 54, No. 1, pp. 34-51, 2006. 